\title{
Effects of a Watermelon Extract Beverage on Canine Lipid Metabolism and Urine Crystals
}

\author{
Sayaka Miyai $^{1, ~ *, ~ T o s h i h a r u ~ H a s h i z u m e ~}{ }^{2}$, Toshio Okazaki ${ }^{1}$ \\ ${ }^{1}$ Yamazaki University of Animal Health Technology, Hachioji, Japan \\ ${ }^{2}$ Hagihara Farm Production Institute, Tawaramoto, Japan \\ Email address: \\ s_miyai@yamazaki.ac.jp (S. Miyai), hashizume@suika-net.co.jp (T. Hashizume), t_okazaki@yamazaki.ac.jp (T. Okazaki) \\ ${ }^{*}$ Corresponding author
}

\section{To cite this article:}

Sayaka Miyai, Toshiharu Hashizume, Toshio Okazaki. Effects of a Watermelon Extract Beverage on Canine Lipid Metabolism and Urine Crystals. Animal and Veterinary Sciences. Vol. 6, No. 5, 2018, pp. 74-79. doi: 10.11648/j.avs.20180605.12

Received: September 26, 2018; Accepted: October 22, 2018; Published: November 15, 2018

\begin{abstract}
Previous report showed that watermelon consumption has an anti-obesity effects in rats. The purpose of this study is to examine the effects on body weight, body fat percentage, serum biochemical data, serum adipokine concentrations (leptin, adiponectin, and resistin), urine specific gravity and sediments when 12 dogs were given watermelon extract beverage instead of water for 3 months. Those data were all assessed before the study period and again at 1.5 and 3 months. Although there were no remarkable changes in most of these parameters, a significant decrease in serum leptin concentrations at 1.5 and 3 months. Calcium oxalate and struvite crystals were observed in the urinary sediment in five dogs; although their urine specific gravities remained $>1.040$ throughout, the number of urinary crystals had decreased by the end of the 3-month period. Morphological components were not found in the urinary sediment of the other five dogs; their urine specific gravities were also $>1.040$ before the study period and at 1.5 months, but these had decreased to $<1.040$ at 3 months. These results suggested that drinking the watermelon extract beverage reduced serum leptin levels and inhibited the formation of urine crystals such as calcium oxalate and struvite crystals in dogs.
\end{abstract}

Keywords: Watermelon, Leptin, Anti-obesity, Anti-urolithiasis

\section{Introduction}

It is known that more than half of domestic dogs are either overweight or obese in the United States [1]. In recent years, obesity-related diseases have been a serious issue for both veterinarians and pet owners [1-3]. Since canine obesity can contribute to the induction of complex physiologic states accompanied by chronic, low-grade inflammation, just as in humans [1-3], many researchers have analyzed the influence of functional foods on animals.

Previous studies have reported that flavonoids extracted from green tea, soy, and fruits improved anti-oxidative index, cytokine and enzyme levels with obesity (e.g., leptin, adiponectin, IL-6 and TNF- $\alpha$ ) [4-6]. In additions, Consumption of these flavonoids resulted in a reduction in fat weight and the recovery to normal levels of adipocyte size in white adipose and hepatic tissue [5,6].

Watermelon (Citrullus lanatus) is a member of the
Cucurbitaceae family of gourds and its flesh contains about $91 \%$ water by weight. The remaining components is a rich source of bioavailable compounds, including lycopene, vitamins $\mathrm{A}$ and $\mathrm{C}$, and the amino acid L-citrulline. Lycopene has been shown to exhibit anti-tumor, anti-inflammatory and anti-obesity effects and L-citrulline is known to have diuretic effects $[7,8]$.

We previously reported that watermelon showed anti-obesity effects in rats, such as a decrease in the plasma concentration of leptin [9]. However, there are no reports about the influence of watermelon consumption on obese dogs. The purpose of this study was to determine how the consumption of a watermelon-based beverage affected canine obesity.

\section{Materials and Methods}

\subsection{Animals and Treatments}

This study was conducted in accordance with the ethical 
guidelines of the Yamazaki Gakuen University Animal Experimentation Committee and was approved by this committee (approval number: H28YAE-No.9).

The study included 12 dogs (two Poodle, one Golden Retriever, two Dachshund, three Chihushus, one Italian Greyhound, one Shih Tzu and two Brussels Griffon) aged 2-9 years. Over a 3-month period, these were freely provided with a watermelon beverage $(150 \mathrm{~mL} / \mathrm{kg} /$ day $)$ instead of water.

\subsection{The Watermelon Beverage}

Watermelon juice was extracted from the flesh around the seeds, adding ethanol at $75 \%$ final concentration. This was then blended for $5 \mathrm{~h}$ and centrifuged at $25,000 \mathrm{~g}$ for $5 \mathrm{~min}$. The supernatant was evaporated at $30^{\circ} \mathrm{C}$ and concentrated to dryness. The beverage was prepared by adding distilled water to the dried extract (which formed $0.6 \%$ by weight) and then ascorbic acid (at approximately $50 \mathrm{mg} / 100 \mathrm{~mL}$ ).

\subsection{Physical Tests}

Each dog's body weight was measured using BTA-SCALE scales (Menix, Tokyo), and its body fat percentage was analyzed using Healthlabo equipment (Kao, Tokyo) and evaluated as one of four patterns (lean, optimal, overweight, and obese) in accordance with the manufacturer's instructions. The dogs' body condition scores were assessed by inspection or palpation on a scale of 1-9 (1-3, lean; 4 and 5, normal; 6 , overweight; 7-9: obese).

\subsection{Serum Analysis}

Blood samples were collected before the study period and after 6 weeks and 3 months of consuming the watermelon beverage. Serum concentrations of urea nitrogen (BUN), creatinine (CRE), glucose (GLU), triglycerides (TG), total cholesterol (TC), and HDL-cholesterol (HDL-C) were analyzed using a Fuji DRI-CHEM dry chemistry analyzer (Fujifilm, Tokyo). Glycoalbumin (GA) measurement was performed by LSI Medience Corp. (Tokyo). ELISA kits were used to measure serum leptin (Millipore Corporation, USA), adiponectin (CircuLexTM; CycLex Co. Ltd., Japan), tumor necrosis factor (TNF)- $\alpha$, and resistin (RayBio, USA) concentrations.

\subsection{Urinary Analysis}

Urine samples were collected by spontaneous urination before the study period and after 6 weeks and 3 months of consuming the watermelon beverage and analyzed using biochemical tests (dipstick and specific gravity test) and microscopic examination (for sediment). The dipstick examination was performed using URIACE-M (Terumo Corp., Tokyo). Specific gravity was analyzed using a refractometer (Erma, Tokyo). Urine sediment was estimated using the method recommended by the Japanese Committee for Clinical Laboratory Standards.

\subsection{Statistical Analysis}

Changes in the analysis data were assessed by analysis of variance and Dunnett's test for multiple comparisons. Statistical analysis was conducted using commercially available software (OMS Statcel 4). $\mathrm{p}<0.05$ was considered statistically significant. Data are presented as mean \pm standard deviation.

\section{Results}

\subsection{Body Weight and Body Fat Percentage}

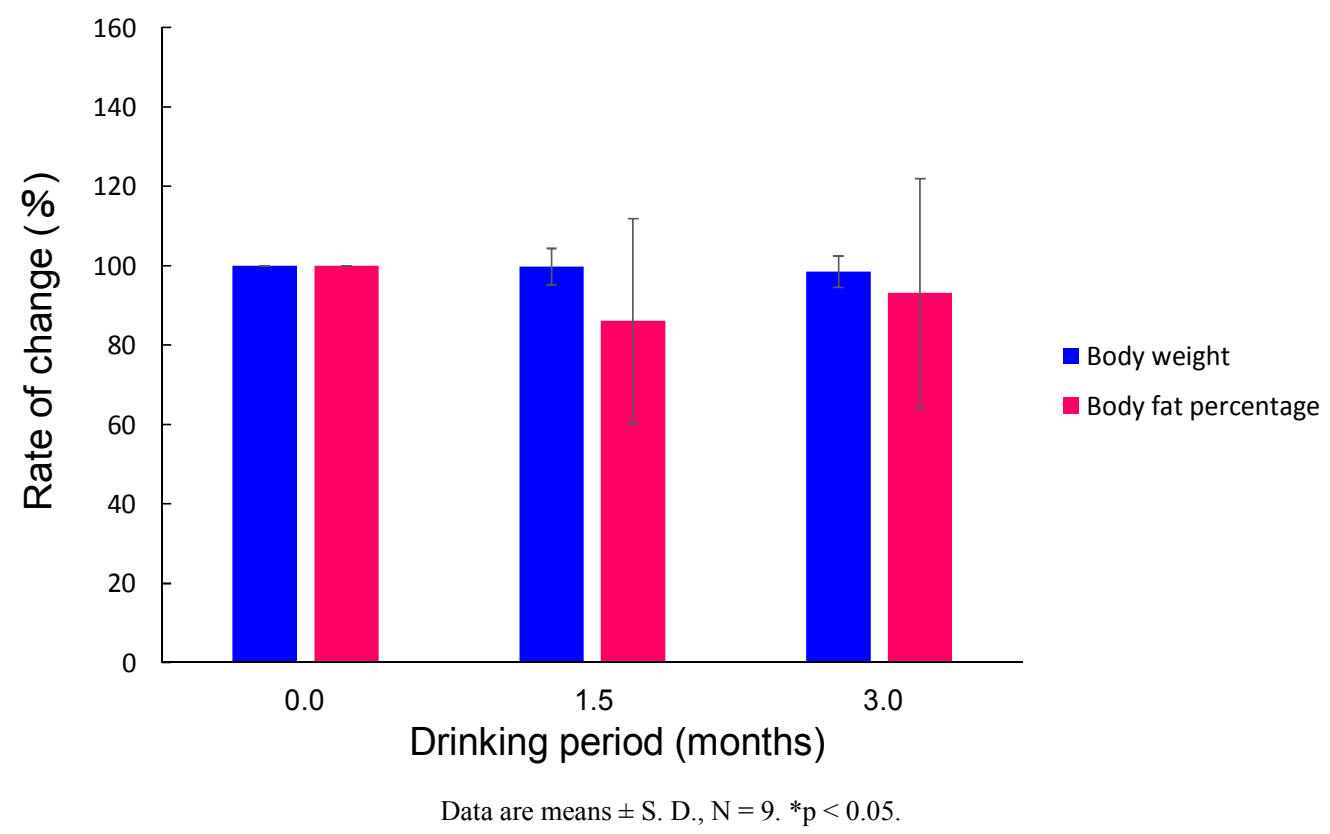

Figure 1. Relative changes in mean body weight and body fat percentage after 1.5 and 3 months of consuming a watermelon-based beverage. The relative changes were calculated by dividing the values by the initial value and expressing this as a percentage. 
The mean body condition scores before, during, and after the 3 -month study period were within the optimum range (before, $4.1 \pm 0.3$; and at 1.5 and 3.0 months, $4.0 \pm 0$ ). However, the body fat percentage varied among the dogs from lean to overweight. There was little change in the dogs' mean body weight over the study period, but their mean body fat percentage changed as follows: before, $30.6 \% \pm 9.3 \%$; 1.5 months, $25.0 \% \pm 6.4 \%$; 3.0 months, $26.0 \% \pm 5.4 \%$. The relative changes in mean body weight and body fat percentage are shown in Figure 1.

\subsection{Serum Biochemical Data}

The serum biochemical values before the study period were

A

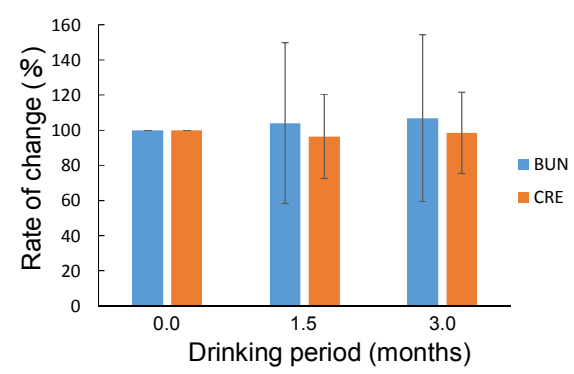

within the normal canine reference ranges given by Fuji Dry Chemistry and LSI Medience (BUN, 9.2-29.2 mg/dL; CRE, 0.4-1.4 mg/dL; GLU, 75-128 mg/dL; TG, 30-133 mg/dL; TC, 111-312 mg/dL; HDL-C, 71-170 mg/dL; and GA: 8\%-14\%). Serum BUN, CRE, GLU, GA, TG, TC, and HDL-C levels did not show any statistically significance change over the study period (Figure 2A-C). TC levels decreased after 1.5 months, but the standard deviation was large and the decrease was not sustained at three months (a relative change of $106.76 \% \pm$ $35.24 \%$; Figure 2C). However, TG levels decreased over the study period (Figure 2C).

B

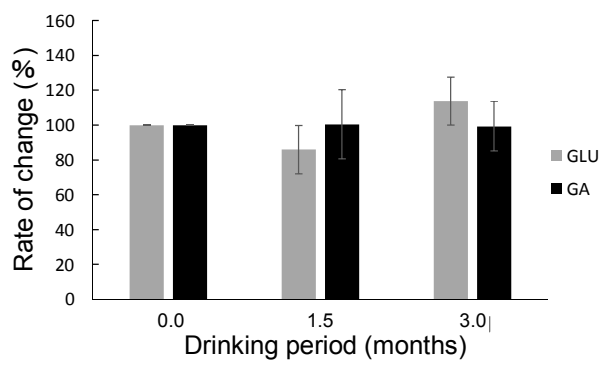

C

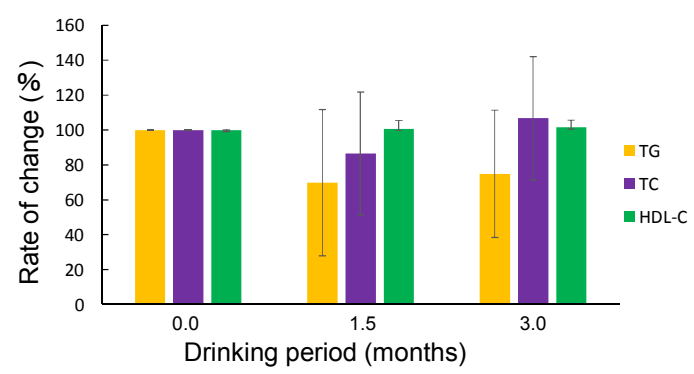

Figure 2. Relative changes in the serum biochemical data over the study period.

(A) Renal dysfunction parameters. BUN, blood urea nitrogen; CRE, creatinine. (B) Carbohydrate metabolism parameters. GLU, glucose; GA, glycoalbumin. (C) Lipid metabolism parameters. TG, triglyceride; TC, total cholesterol; HDL-C, high-density lipoprotein cholesterol.

Data are means \pm S. D., $\mathrm{N}=9 .{ }^{*} \mathrm{p}<0.05$.

\subsection{Serum Adipokine Concentrations}

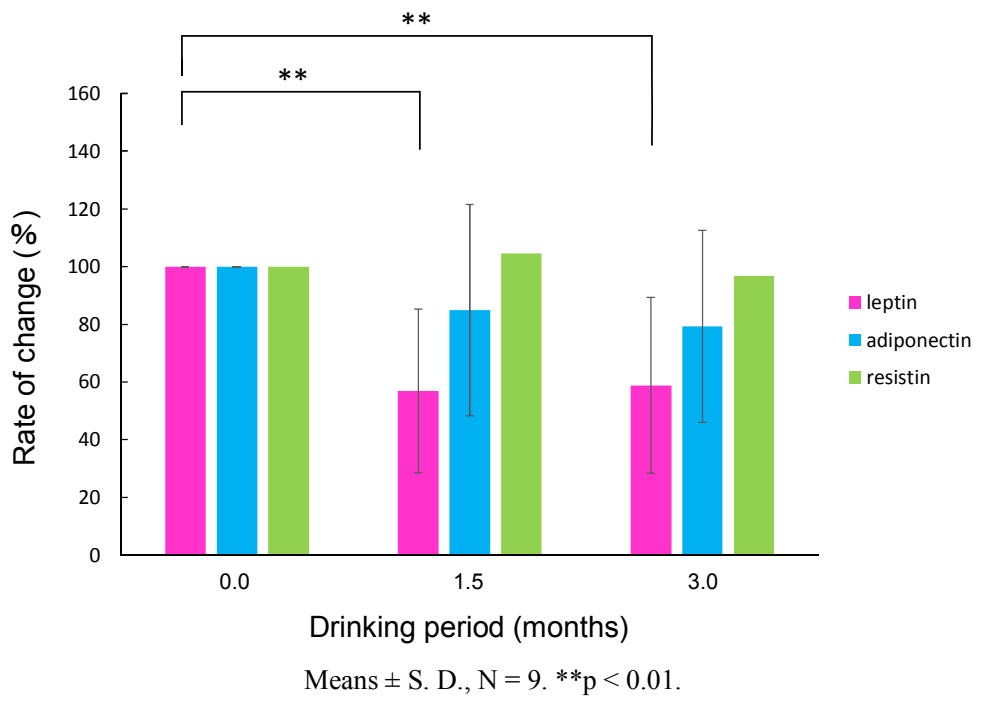

Figure 3. Relative changes in the serum adipokine (leptin, adiponectin and resistin) concentrations over the study period. 
Mean values for the adipokine concentrations before the study and at 1.5 and 3 months were as follows: leptin, $5.56 \pm$ $1.92,3.19 \pm 1.92$, and $3.49 \pm 2.66 \mathrm{ng} / \mathrm{mL}$; adiponectin, $4.42 \pm$ $4.26,2.90 \pm 1.83$, and $2.69 \pm 1.44 \mathrm{ng} / \mathrm{mL}$; and resistin, $5.23 \pm$ $2.27,5.45 \pm 2.59$, and $4.89 \pm 2.07 \mathrm{ng} / \mathrm{mL}$. TNF- $\alpha$ was not detected (data not shown). Leptin levels showed significant decreases, but adiponectin and resistin levels did not change significantly (Figure 3).

A

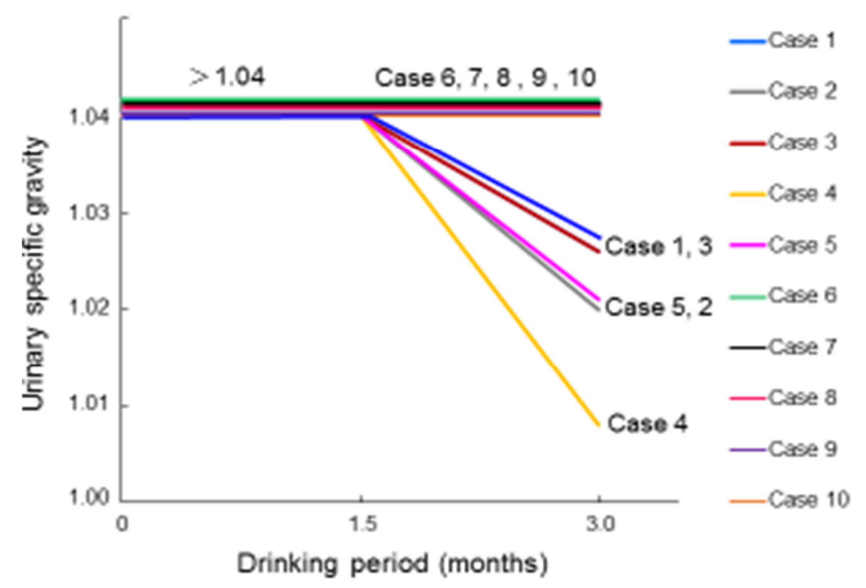

C

\subsection{Urine Specific Gravity and Sediment}

All of the urine specific gravity values before and after 1.5 months were $>1.040$. However, after 3 months, the urine specific gravity had decreased in 5 of the 10 dogs (Case 1, 1.026; Case 2, 1.020; Case 3, 1.026; Case 4, 1.008; Case 5, 1.021) (Figure 4A).

\section{B}

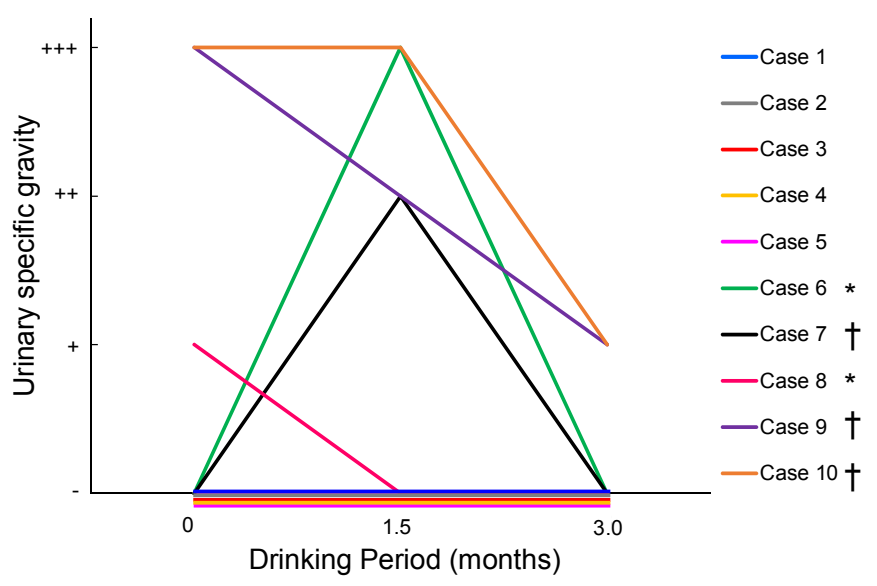

Case 6: *Calcium Oxylate crystal
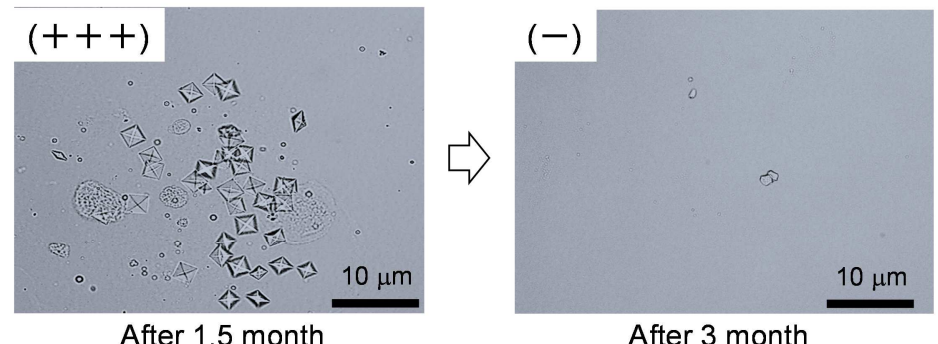

Case 10: $\uparrow$ Struvite crystal
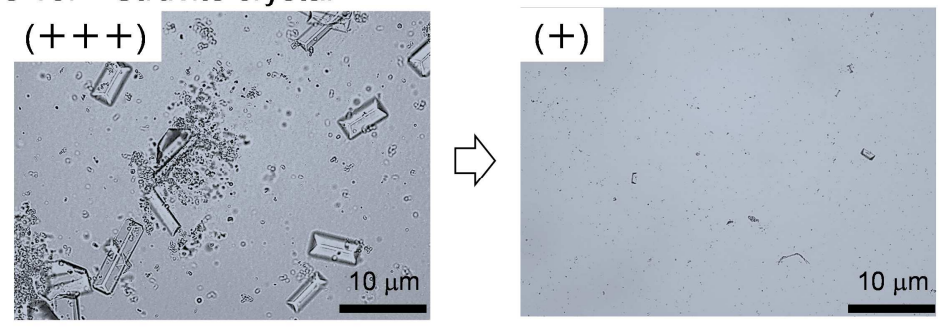

Before and after 1.5 month

After 3 month

Figure 4. Urine specific gravity and urine crystals (calusium oxalate or struvite) analyses.

(A) Changes in urine specific gravity values for individual dogs during the study period. $\mathrm{N}=10$. (B) Changes in urine crystals for individual dogs during the study period. $\mathrm{N}=10$. -; No crystals observed, +; a small number of crystals observed, ++; some of crystals observed, +++; a large number of crystals observed, *; calcium oxalate crystal, $\uparrow$; struvite crystal. (C) Microscopic photographs of urine calcium oxalate and struvite crystals for two characteristic cases.

A small number of squamous epithelial cells or bacteria were present before and at 1.5 months in Cases 1-5, all within the normal range. However, these cases showed no morphological components after 3 months (data not shown). Calcium oxalate crystals were detected in the urinary sediment of Cases 6 and 8 before the study period and at 1.5 months (Figure 4B). Struvite crystals were observed in the urinary sediment of Cases 7, 9, and 10 at each time point. The crystal levels in Cases 6-10 were lower at the end of the study period than at the start (Figure 4B). Figure 4C shows microscopy 
photographs of urine crystals (calcium oxalate and struvite) for two characteristic cases.

\section{Discussion}

This study investigated the effects of drinking a watermelon-based beverage over a three-month period on the body weight, body fat percentage, serum biochemical data, and serum adipokine data of dogs. No changes were seen in most of these parameters; however, there was a slight decrease in serum TG and a significant change in leptin levels over the study period, although adiponectin and resistin levels did not change.

The small decrease in serum TG suggested that the watermelon extract included a compound or compounds that affected the lipid metabolism of dogs. In a recent study, the feeding with watermelon improved serum lipid profiles such as TG through changes in the expression of genes related to lipid metabolism [10]. However, it remained to be elucidated which compounds in watermelon have the effect on lipid metabolism.

Adipokines, including leptin, adiponectin and resistin, are biologically active substances that are secreted mainly from mature adipose tissue, playing important roles in the regulation of the lipid and carbohydrate metabolism [11, 12]. Because leptin primarily works as a fasting hormone thorough the regulation of food intake and energy consumption, plasma leptin levels are quantitative markers of obesity in humans, dogs and rodents $[13,14]$. It is known that circulating leptin levels correlate positively with adipose tissue mass and body condition scores [14]. It has been suggested the decrease of the number of adipose cells from the reductions of leptin level over the study period. However, we were unable to confirm the relationship between serum leptin levels and the body fat percentage values. Because the body condition scores of all the dogs used in the study were within the normal range (from 4 to 5 in the 9-point system), it was difficult to confirm any changes in body weight, body fat percentage, and the serum biochemical data. It is known that adiponectin contributes to increased insulin sensitivity, reduced blood glucose and reduced tissue TG levels in the liver and muscle [14]. Resistin promotes inflammation and regulates the secretion of proinflammatory cytokines such as TNF- $\alpha$ and interleukin- 6 (IL-6) [11-15]. However, there was only a slight change in adiponectin and no change in resistin over the study period. Although it has been assumed that adiponectin levels correlate with the number or size of adipose cells, changes in the number or size of adipose cells were not examined in the present study. It would be valuable to investigate these aspects in future study.

Mentioned earlier, watermelons contain various flavonoids, such as lycopene and $\beta$-carotene. Flavonoids in the watermelon-based beverage administered to the dogs may have reduced the TG concentrations via an effect on the lipid metabolism such as stimulation of fatty acid oxidation or the inhibition of lipid storage. These effects may have resulted in the lower leptin concentration through a decrease in adipocytes.

Effect of the beverage on urinary data was examined. We confirmed urine crystals (calcium oxalate and struvite) in the urinary sediments in five of the dogs, whose urine specific gravities remained at more than 1.040 throughout the study period (Figure $4 \mathrm{~A}$ and $\mathrm{B}$ ). In all of these dogs, the number of urinary crystals decreased by the end of three months (Figure 4B). We were unable to identify morphological components in the urinary sediment of the other five dogs, whose urine specific gravities were $>1.040$ before and at 1.5 months, but $<1.040$ at 3 months (Figure 4A). These results suggest that drinking a watermelon-based beverage over three months may reduce urine specific gravities and the number of urine crystals.

Urinary stones such as calcium oxalate and struvite (magnesium ammonium phosphate hexahydrate) are a commonly experienced clinical problem in dogs; such stones may be correlated with obesity in humans and canines [16, 17]. It is known that urinary stones form through a complex process connected with supersaturation, nucleation, aggregation, and the growth and retention of crystals within the renal tubules $[16,17]$. It is possible to reduce the formation of urinary stone by increasing water intake to dilute the urine, consuming formula diets to restrict mineral intake and performing physical exercise. Although dogs do not freely drink enough water, it is difficult for owners to give much water to their dogs. It may therefore be useful to provide a watermelon-based beverage for dogs that do not drink water and suffer from urolithiasis. However, in this study it took 3 months after the start of administration of the beverage for the dogs' urine specific gravity and the number of urine crystals to decrease. It may therefore take about 2 months of drinking such a watermelon beverage constantly for the dogs to experience the benefits.

Recently, bioactive substances extracted from plants, such as polyphenols and flavonoids, have anti-urolithiatic effects by increasing urine volume [18-20]. Watermelon contains useful components such as L-citrulline, which has a diuretic effect. The results of the present study suggested that administration of the had a diuretic effect, promoting spontaneous water intake, reducing urine specific gravities and inhibiting the crystallization of urine stones (calcium oxalate and struvite crystals). However, it has recently been reported that L-citrulline in watermelons induced a significant reduction of food intake by appetite suppression, involving leptin signaling [16]. Thus, the L-citrulline in watermelons may also have an effect on obesity [21].

Recently, the morbidity rates for obesity and urine stones in dogs have increased, leading owners and veterinarians to pay attention to the prevention of obesity and renal disorders. Further studies are needed to identify the effective compounds in the watermelons-based beverage that reduce serum leptin levels and inhibit the formation of urinary stones, as well as to elucidate the mechanisms by which the active components in the beverage induce the effects on adipocytes. 


\section{Conclusions}

This study revealed that drinking the watermelon extract beverage reduced serum leptin levels and inhibited the formation of urine crystals such as calcium oxalate and struvite crystals in dogs.

\section{Acknowledgements}

We would like to thank all the Yamazaki University of Animal Health Technology stuffs who prepared the dogs for our experiment. This work is supported by a grant for Hagihara Farm Production Institute. Finally, we are grateful to the referees and Enago for useful comments and calibration English.

\section{References}

[1] Li Q, Lauber CL, Czarnecki-Maulden G, Pan Y and Hannah SS 2017. Effects of the dietary protein and carbohydrate ratio on gut microbiomes in dogs of different body conditions. MBio. Vol. 8, No. 1, pii: e01703-e01716, doi: 10.1128/mBio.01703-16.

[2] Baric Rafaj R, Kules J, Marinculic A, Tvarijonaviciute A, Ceron J, Mihaljevic Z, Tumpa A and Mrljak V, 2017. Plasma markers of inflammation and hemostatic and endothelial activity in naturally overweight and obese dogs. BMC Vet Res. Vol. 13, No. 13, doi: 10.1186/s12917-016-0929-8.

[3] Radakovich LB, Truelove MP, Pannone SC, Olver CS and Santangelo KS, 2017. Clinically healthy overweight and obese dogs differ from lean controls in select $\mathrm{CBC}$ and serum biochemistry values. Vet Clin Pathol. Vol. 46, No. 2, 221-226, doi: $10.1111 /$ vep. 12468 .

[4] Xu Y, Zhang M, Wu T, Dai S, Xu J and Zhou Z, 2015. The anti-obesity effect of green tea polysaccharides, polyphenols and caffeine in rats fed with a high-fat diet. Food Funct. Vol. 6, No. 1, pp: 297-304, doi: 10.1039/c4fo00970c.

[5] Lee EH, Son WC, Lee SE and Kim BH, 2013. Anti-obesity effects of poly- $\gamma$-glutamic acid with or without isoflavones on high-fat diet induced obese mice. Biosci Biotechnol Biochem. Vol. 77, No. 8, pp: 1694-1702, doi:10.1271/bbb.130253.

[6] Jo YH, Choi KM, Liu Q, Kim SB, Ji HJ, Kim M, Shin SK, Do SG, Shin E, Jung G, Yoo HS, Hwang BY and Lee MK, 2015. Anti-obesity effect of 6, 8-diprenylgenistein, an isoflavonoid of cudrania tricuspidata fruits in high-fat diet-induced obese mice. Nutrients. Vol. 7, No. 12, 10480-10490, doi: $10.3390 /$ nu7125544.

[7] Shanely RA, Nieman DC, Perkins-Veazie P, Henson DA, Meaney MP, Knab AM and Cialdell-Kam L, 2016. Comparison of watermelon and carbohydrate beverage on exercise-induced alterations in systemic inflammation, immune dysfunction, and plasma antioxidant capacity. Nutrients. Vol. 8 , No. 8, pii: E518, doi: 10.3390/nu8080518.

[8] Tamburini E, Costa S, Rugiero I, Pedrini P and Marchetti MG, 2017. Quantification of lycopene, $\beta$-carotene, and total soluble solids in intact red-flesh watermelon (citrullus lanatus) using on-line near-infrared spectroscopy. Sensors (Basel). Vol. 17, No. 4, pii: E746, doi: 10.3390/s17040746.
[9] Okazaki T, Hashizume T, Suzuki M and Ogawa Z, 2014. Anti-obesity effects of watermelon extract on rats fed high-fat diet. Journal of Pet Animal Nutrition. Vol. 17, No. 1, pp. 13-18, doi: https://doi.org/10.11266/jpan.17.13.

[10] Hong MY, Hartig N, Kaufman K, Hooshmand S, Figueroa A and Kern M, 2015. Watermelon consumption improves inflammation and antioxidant capacity in rats fed an atherogenic diet. Nutr Res. Vol. 35, No. 3, pp: 251-258, doi: 10.1016/j.nutres.2014.12.005.

[11] Kim HS, Kang JH and Jeung EB, 2016. Yang MP. Serum concentrations of leptin and adiponectin in dogs with myxomatous mitral valve disease. J Vet Intern Med. Vol. 30, No. 5, pp: 1589-1600. doi: 10.1111/jvim.14570.

[12] Ismail MM, Abdel Hamid TA, Ibrahim AA and Marzouk H, 2017. Serum adipokines and vitamin D levels in patients with type 1 diabetes mellitus. Arch Med Sci. Vol. 13, No.4, pp: 738744. doi: 10.5114/aoms.2016.60680.

[13] Lee S, Kweon OK and Kim WH, 2017. Increased Leptin and Leptin Receptor Expression in Dogs With Gallbladder Mucocele. J Vet Intern Med. Vol. 31, No.1, pp: 36-42. doi: 10.1111/jvim. 14612 .

[14] Park HJ, Lee SE, Oh JH, Seo KWand Song KH, 2014. Leptin, adiponectin and serotonin levels in lean and obese dogs. BMC Vet Res. Vol. 13, No. 10, pii: 113. doi: 10.1186/1746-6148-10-113.

[15] Ren Y, Wan T, Zuo Z, Cui H, Peng X, Fang J, Deng J, Hu Y, Yu S, Shen L, Ma X, Wang Y and Ren Z, 2017. Resistin increases the expression of NOD2 in mouse monocytes. Exp and Ther Med. No. 13, Vol. 5, pp: 2523-2528. doi: 10.3892/etm.2017.4288.

[16] Calabro S, Tudisco R, Bianchi S, Grossi M, De Bonis A and Isabella Cutrignelli M, 2011. Management of struvite uroliths in dogs. Br Journal Nutr. Vol. 106, No. 1, pp: 191-193. doi: $10.1017 / \mathrm{S} 0007114511000882$.

[17] Bartges JW and Callens AJ, 2015. Urolithiasis. Vet Clin North Am Small Anim Pract. Vol. 45, No. 4, pp: 747-768. doi: 10.1016/j.cvsm.2015.03.001.

[18] Yadav M, Gulkari VD and Wanjari MM, 2016. Bryophyllum pinnatum leaf extracts prevent formation of renal calculi in lithiatic rats. Anc Sci Life. Vol. 36, No. 2, pp: 90-97. doi: 10.4103/asl. ASL 9016.

[19] Das P, Kumar K, Nambiraj A, Rajan R, Awasthi R, Dua K and M H, 2017. Potential therapeutic activity of Phlogacanthus thyrsiformis Hardow (Mabb) flower extract and its biofabricated silver nanoparticles against chemically induced urolithiasis in male Wistar rats. Int J Biol Macromol. Vol. 103, pp: 621-629. doi: 10.1016/j.ijbiomac.2017.05.096.

[20] Velu V, Das M, Raj N AN, Dua K and Malipeddi H, 2017. Evaluation of in vitro and in vivo anti-urolithiatic activity of silver nanoparticles containing aqueous leaf extract of Tragia involucrata. Drug Deliv and Transl Res. Vol. 7, No. 3, pp: 439449. doi: 10.1007/s13346-017-0363-x.

[21] Kudo M, Yoshitomi H, Momoo M, Suguro S, Yamagishi Y and Gao M, 2017. Evaluation of the effects and mechanism of L-Citrulline on anti- obesity by appetite suppression in obese/diabetic KK-Ay mice and high-fat diet fed SD rats. Biol Pharm Bull. Vol. 40, No. 4, pp: 524-530. doi: 10.1248/bpb.b16-01002. 